International Journal of Engineering \& Technology, $7(1.5)(2018) 135-140$
International Journal of Engineering \& Technology
SPC
Website: www.sciencepubco.com/index.php/IJET
Research paper

\title{
Glaucoma detection using cup to disc ratio and artificial neural networks
}

\author{
Gayathri R. ${ }^{1 *}$, Rao P. V. ${ }^{2}$ \\ ${ }^{1}$ Research Scholar, Electronics Engineering, Jain University, Bangalore, Karnataka, India \\ ${ }^{2}$ Professor, PG Coordinator, Vignana Bharathi Institute of Technology, Hyderabad, India \\ *Corresponding author E-mail: gayathrir855@gmail.com
}

\begin{abstract}
Now-a-days, the most commonly predicted eye disease in human beings is glaucoma; loss of vision gradually may turn into blindness. Advanced image handling methods empower osteopathic specialist to distinguish and treat a few eye infections like diabetic retinopathy and glaucoma. The pressure in the optic nerve of the eye may lead to get affected by glaucoma, which is most regular reason for visual deficiency of the peoples, if not treated appropriately at early stage. The main objective of this paper is the detection of glaucoma and classifies the disease based on its severity using artificial neural network. In this paper mainly focused on pre -processing of retinal fundus images for improving the quality of detection and easy to further handling. The simulation results to obtain using MATLAB for the better accuracy in detecting glaucoma for abnormality using Cup to Disc ratio of retinal fund us images.
\end{abstract}

Keywords: Pre-Processing, Cup-to-Disc, Glaucoma, Morphological operations, Segmentation, Artificial Neural Networks.

\section{Introduction}

The two noteworthy reasons for the infection are the Diabetic Retinopathy (DR) and Glaucoma for retinal issue which prompts vision loss if not treated properly at an early stage. Extremely basic is early screening is done as such that the patient is out of risk. The Osteopathic specialists are going to either take fundus images or OCT images for location and conclusion of the sickness [19]. The optic nerve of the eye is the disease of the glaucoma which may happen and ends up noticeably extreme after some time. Due to more pressure in the eye glaucoma will happen. Glaucoma is one of the acquired sicknesses and not demonstrates any symptoms in early stage. The pressure that developed on eye increments are the intraocular pressure and harms the optic nerve where the pictures are transmitted to the brain. It is one of the optic neuropathy by unmistakable changes and described in the optic nerve head and visual field[1]. By constantly harming optic nerve glaucoma will bring about lasting Vision Loss and can bring about aggregate perpetual visual deficiency inside a couple of years if not medicated properly. Glaucoma won't demonstrate any side effects or symptoms in early stage from this increased pressure. The imperative and testing perspectives are the essential open angle glaucoma (OAG) management. It is essential to recognize and limit the danger of the visual misfortune in highlight. For prior finding of glaucoma the best technique included is the combination of structural and functional tests [5]. In this method Cup to Disc ratio (CDR) is found to recognize at the early stage and arranged the seriousness of the infection [15]. In another strategy Structural and energy features are utilized then examined and ordered for glaucomatous image [5]. Here image segmentation algorithm is used to extract cup and disc features and detecting the glaucoma disease [5]. Both normal and glaucoma fundus images of retina [1] are shown in figure.1. In this proposed work a novel algorithmic based approach for glaucoma detection incorporating both Cup to Disc Ratio and Artificial Neural networks principles for identification of abnormalities from fundus images. The research work of this paper is organized as follows; 2. Artificial Neural Networks: 3. Methodology: 4. Experiment Results: 5. Conclusion.

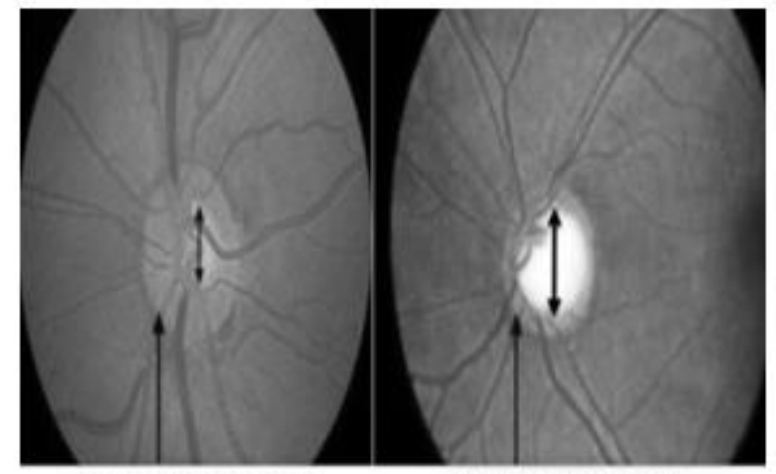

Nomal optic nerve head

Glaucomatous cupping

Fig. 1: Normal and Glaucomatous status of fundus images.

\section{Artificial Neural Networks}

A neuron (neurone or nerve cell) is a cell that carries electrical impulses. Neurons are mainly motivated for processing Neurons are the basic elements of the nervous system and plays an important role in functioning of the brain is shown in Figure 2. 


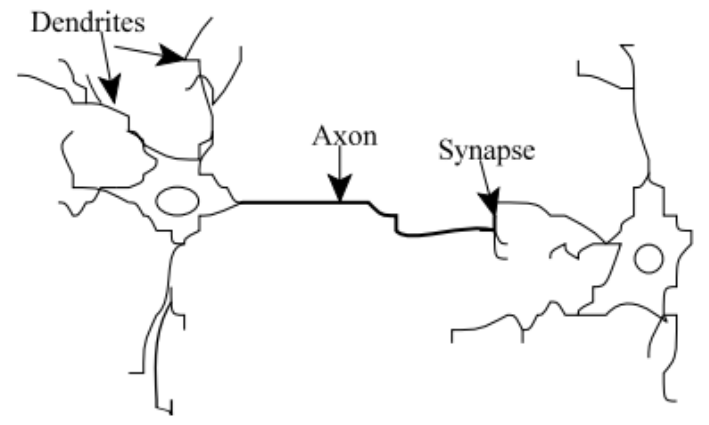

Fig. 2: Fundamentals of Natural neurons

Each neuron is composed of three parts made of a cell body-soma, dendrites and axon. Dendrites and axons are nerve fibers. There are about 100 billion neurons in the human brain, which comprises roughly $10 \%$ of all brain cells. The neurons are supported by glial cells and astrocytes. Neurons are connected to one another and tissues[12]. They do not touch and instead form tiny gaps called synapses. These gaps can be chemical synapses or electrical synapses and pass the signal from one neuron to the next.One of the grungy electronic models in perspective of the neural structure of the mind is the Artificial Neural Networks (ANN)[13]. An artificial neuron is a mathematical function conceived as a model of biological neurons, a neural network. Artificial neurons are elementary units in an artificial neural network, is shown in Figure.3.

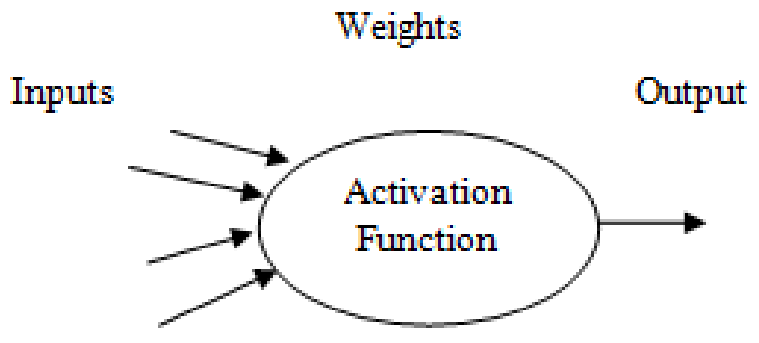

Fig. 3: An Artificial Neuron

Actually, the brain on an extremely fundamental level picks up [11]. This brain duplicate in like manner ensures a less particular way to deal with make machine courses of action. 'Unnatural neurons' are one kind of framework sees the nodes. These are called unnatural neural systems. An unnatural neuron is a computational model unprecedented in the regular neurons [18]. Membrane of the neuron gets the common neurons that are situated on the neuron transmitters through signals. At the point when signal is strong the neuron become active and it radiates a signal though the axon. Next the same signal sent to other synapse and may switch to other. Here, the complexity is especially high when modelling with unnatural neurons. The neurons are multiplied by weights and then it is observed continuously by scientific operation to activate the other neuron. By modifying the weights, the outputs are obtained for each input. The way of this recurring process for changing or adjusting the weights is called learning [13]. The quantity of sorts of ANNs and their uses is high. Higher the weight powerful is input that is multiplied. Negative weights additionally there however these will provide to the inputs and turn to uncomfortable for analysing[12]. Whenever these data of more than hundreds or thousands of neurons, it is very complicate and tedious method by hand to discover all the required significant weights. So in this research work, we have proposed a novel algorithmic based approach that adjusts and provides suitable weights which can easily identify the abnormality of fundus images with more accuracy.

The fundamental approach of an artificial neural system is shown in Figure 4. Artificial neural system is an interconnected gathering of nodes to the limitless system of neurons in a brain [16]. Here, every round about node speaks to an artificial neuron and an arrow represents to an association from the output of one neuron to the input of another.

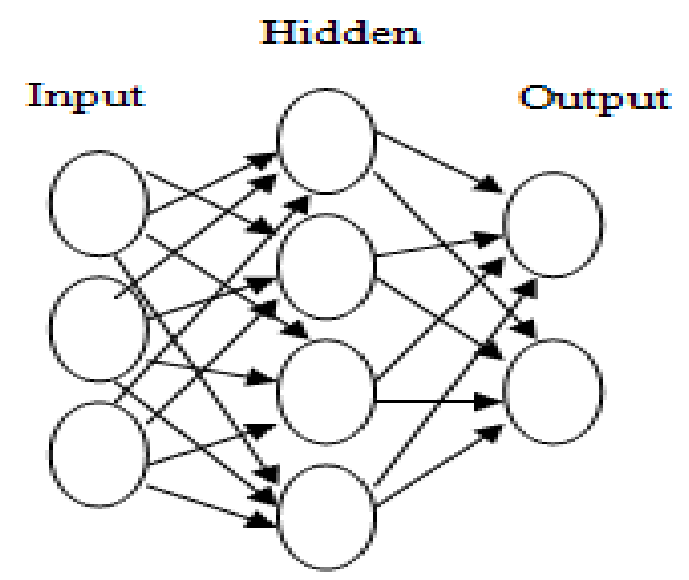

Fig. 4: An artificial Neural Network

\section{Methodology}

The proposed methodology for detection of glaucoma of fundus images is shown in Figure 5. A fundus image of database which is made in JEPG format is created [14]. Initial phase in the process is pre-processing step. The term of the operations of pre-processing diminish the level of pictures on abstraction [5]. Pre-processing is intend to change of the picture or image information that stifles undesired distortion features relevant for next investigation and processing [1]. Here the image is resized to a color space changes and specific size in view of necessity [4]. Pre-processed yield is connected to the segmentation procedure to concentrate Cup and Disc shapes from the input picture. Image segmentation should be possible for required measure of area for specific application [6].

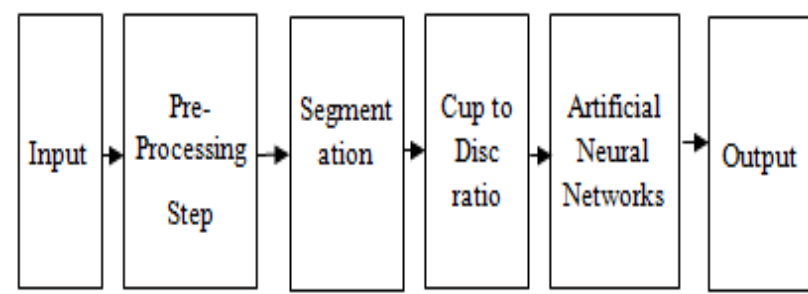

Fig. 5: Block diagram of the proposed method for glaucoma detection

The segmentation is taken from estimations and picture might be grey level, color, texture, depth or motion. The CTD is figured from the areas of optic cup and optic disc[1]. CTD proportions are ordered into stages like typical, first stage, second stage and third stage. The CTD proportions are connected to the Artificial Neural Networks for confirming the performance and error histogram. Database comprises of thirty retinal pictures. The images are put away in JPEG format file(.jpg). Initial step is to peruse picture and change over it to NTSC color space to concentrate green divert in the image. RGB2NTSC charge used to change over the RGB qualities to NTSC color space [20]. RGB to NTSC (rgbmap) changed over picture green channel is separated from it and is connected to the morphological operation 


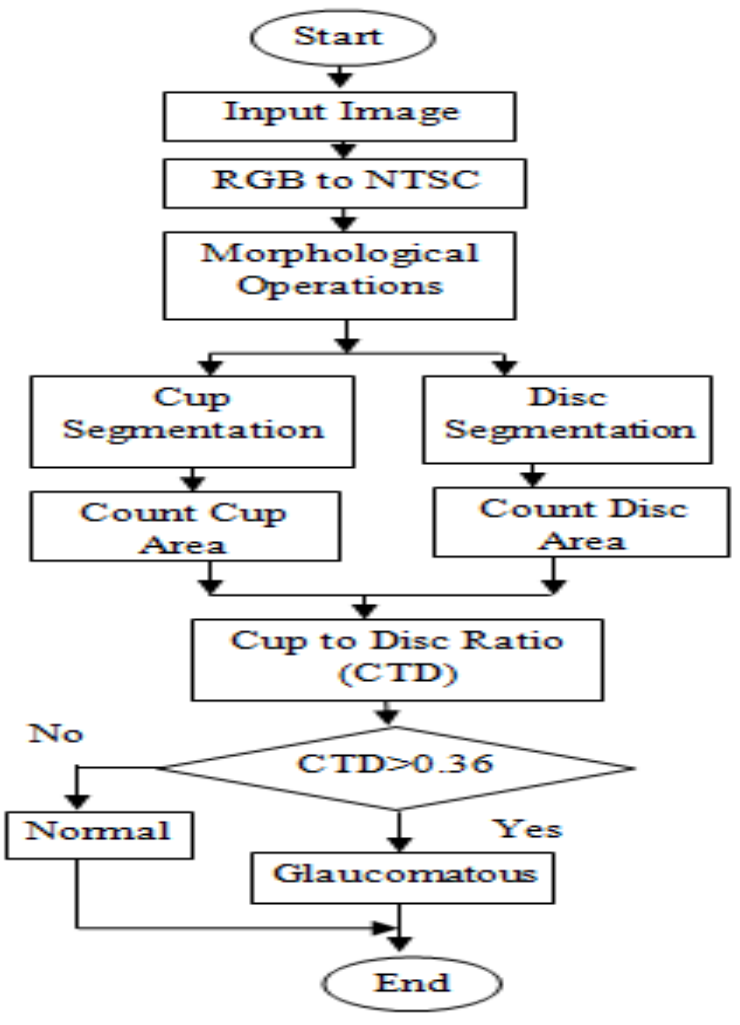

Fig. 6: Flow chart of Glaucomatous or Normal Image

Figure 6 shows the flow chart of Glaucomatous or Normal Image. The utilization of morphological operations is to assume a key role in machine vision and object recognition [14]. Morphological operations are utilized to comprehend the structure of image. Morphological systems test aimage with a little shape or layout called structuring element. The structuring component is situated at all conceivable areas of the picture compared with neighborhood pixels [11]. The morphological operation yield is connected to the segmentation procedure. Here watershed segmentation is utilized to portion the cup and disc area of the eye. The watershed adds to improve the numerical outcomes for picture segmentation problems. The segmented cup and disc which consists of white pixels by counting those pixels calculate the area of the cup and disc [8]. Calculate the CDR and compare the ratio for normal cup to disc ratio. For normal eye CDR is 0.3 , if the input image CDR exceeds more than 0.3 , then it is to be considered as an image affected by glaucoma. After finding normal or abnormality of fundus images (glaucoma), then it is fed to artificial neural networks for further processing.

The flow chart of artificial neural system (ANN) is shown in Figure.7. The CDR of fundus database values are applied to the ANN. ANN Architecture is three layers which comprises of input, hidden and output layer[7]. Contribution to the ANN is two and yield is four i.e. database is characterized to four classes. After executing the ANN architecture performance, error histogram and regression plots are obtained. After ANN classify CTD ratios to four stages: normal, $1^{\text {st }}, 2^{\text {nd }}$ and $3^{\text {rd }}$ or advanced.

The performance plot of the ANN show exhibits that mean square error turns out to be less than as the quantity of epochs is increasing. The test set error and validation set mistake has equivalent attributes and no major over fitting has occurred close to some epoch where the best performance execution occurred[19]. The error histogram plot is to present further authentication of network performance. It points towards outliers, which are the perfect fit indicates that the data should fall along line (slope is close to 1), means network output is equal to targets. The Regression value demonstrates the connection between the yield or output and targets [15]. In the event that regression is closer to one then it demonstrates the correct straight connection amongst yield and targets. In the event that regression esteem is closer to zero then it demonstrates the no straight connection amongst yield and targets[20].

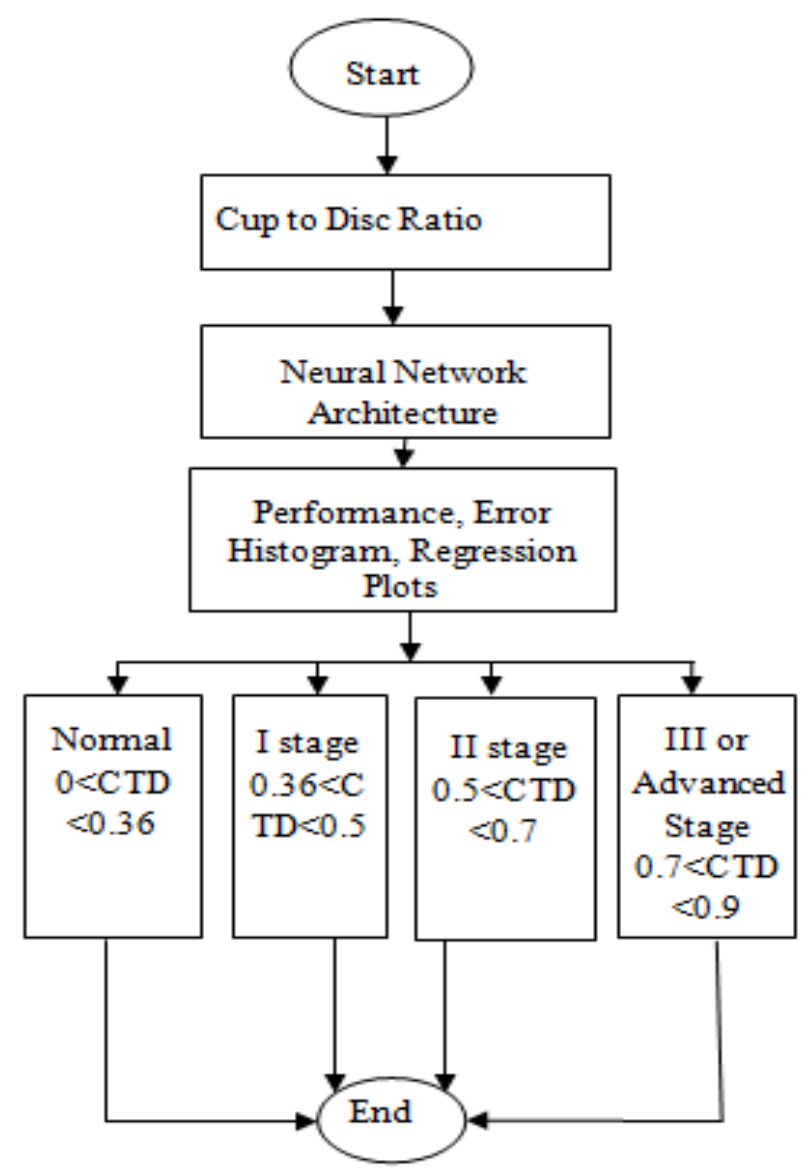

Fig. 7: Flow chart of ANN

\section{Experiment Results}

Table 1: Cup to disc ratio for normal image dataset

\begin{tabular}{|c|c|c|c|c|}
\hline Sr. No. & Cup Area & Disc Area & $\begin{array}{c}\text { Cup to } \\
\text { Disc ratio } \\
\text { (CTD) }\end{array}$ & $\begin{array}{c}\text { Previous } \\
\text { technique } \\
\text { (CTD) }\end{array}$ \\
\hline 1 & 569.375 & 1141.25 & 0.4989 & 0.4932 \\
\hline 2 & 557.750 & 951.000 & 0.5865 & 0.5822 \\
\hline 3 & 558.125 & 905.375 & 0.6145 & 1.0569 \\
\hline 4 & 653.250 & 1184.50 & 0.5515 & 0.5432 \\
\hline 5 & 324.750 & 820.250 & 0.3959 & 0.3980 \\
\hline 6 & 363.375 & 903.000 & 0.4024 & 0.4025 \\
\hline 7 & 465.750 & 815.875 & 0.5708 & 0.5688 \\
\hline 8 & 526.125 & 807.750 & 0.6513 & 0.6653 \\
\hline 9 & 569.250 & 1134.375 & 0.5018 & 0.4932 \\
\hline 10 & 517.625 & 875.750 & 0.5911 & 0.5822 \\
\hline 11 & 366.625 & 903.500 & 0.4058 & 0.3892 \\
\hline 12 & 285.875 & 695.625 & 0.4109 & 0.3542 \\
\hline 13 & 313.750 & 800.000 & 0.3922 & 0.3414 \\
\hline 14 & 413.250 & 767.125 & 0.5387 & 0.4438 \\
\hline 15 & 428.625 & 956.625 & 0.4480 & 0.4559 \\
\hline
\end{tabular}

Table. 1 and Table. 2 shows the Cup to Disc ratio for normal image dataset and abnormal image dataset. Here we comparethe present technique to the previous technique. In the previous technique 
thresholding the region having uniform pixel values are grouped which gives the measurement of the segmented cup area[6].To find the disc area of input image with different threshold values are applied and related to the cup areas. After finding disc area keep disc area as constant for all the different images of the eye and cup area varied by applying threshold values for different regions[21]. In the present technique the author measures the cup area and disc area keeping the structureing element on it and do the segmentation for required region, Which gives the more accurate result compare to previous result[21]. Here the simulation results gives the better accuracy compared to the previous work. Figure 8 gives a snapshot of the graphical user interface (GUI) to identify the glaucoma using the fundus pictures.

Table 2: Cup to disc area for abnormal image dataset

\begin{tabular}{|c|c|c|c|c|}
\hline $\begin{array}{l}\text { Sr. } \\
\text { No. }\end{array}$ & Cup Area & Disc Area & $\begin{array}{l}\text { Cup to } \\
\text { Disc ratio }\end{array}$ & $\begin{array}{c}\text { Previous } \\
\text { technique } \\
\text { (CTD) }\end{array}$ \\
\hline 1 & 269.25 & 835.875 & 0.3222 & 0.4010 \\
\hline 2 & 367.75 & 1133.875 & 0.3243 & 0.3822 \\
\hline 3 & 264.875 & 759.625 & 0.3487 & 0.5258 \\
\hline 4 & 261.875 & 749 & 0.3298 & 0.5252 \\
\hline 5 & 262.885 & 753 & 0.3258 & 0.5256 \\
\hline 6 & 286 & 924.875 & 0.3092 & 0.3945 \\
\hline 7 & 287.625 & 944 & 0.30469 & 0.3052 \\
\hline 8 & 284.875 & 1044.75 & 0.2727 & 0.3229 \\
\hline 9 & 273.25 & 1264.625 & 0.2161 & 0.3696 \\
\hline 10 & 216.75 & 939.625 & 0.23068 & 0.4436 \\
\hline 11 & 293.875 & 1192.50 & 0.2464 & 0.3784 \\
\hline 12 & 206.5 & 982.25 & 0.2010 & 0.3298 \\
\hline 13 & 187.625 & 1107.375 & 0.1694 & 0.3320 \\
\hline 14 & 199.75 & 1057 & 0.1889 & 0.2796 \\
\hline 15 & 205.875 & 707 & 0.2912 & 0.2914 \\
\hline
\end{tabular}

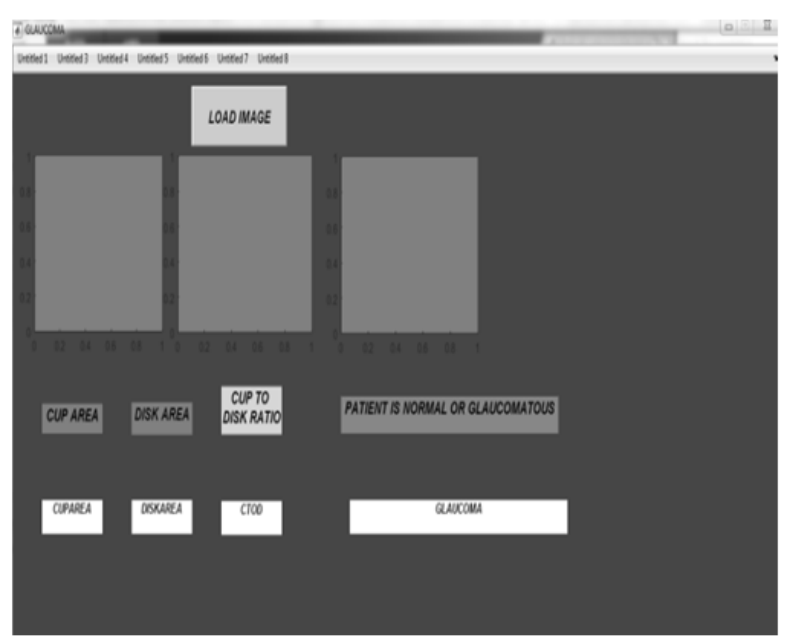

Fig. 8: GUI window for glaucoma detection

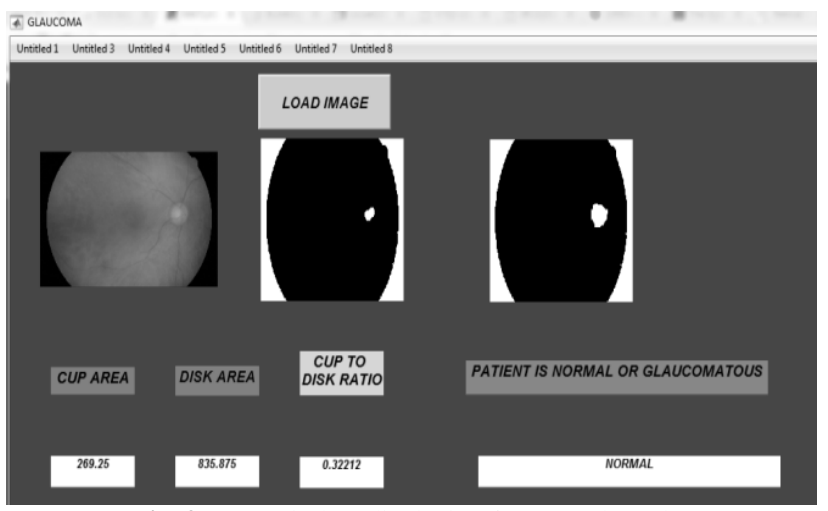

Fig. 9: GUI window displaying for normal results

A normal image is chosen the database connected to GUI window and the outcomes are shown as appeared in the Figure.9. The outcomes Cup area, Disc area and Cup to Disc ratio are 269.25, 836.876 and 0.32212 respectively. A glaucomatous image is chosen from the database connected to GUI window and the GUI representation is Figure 10[19].

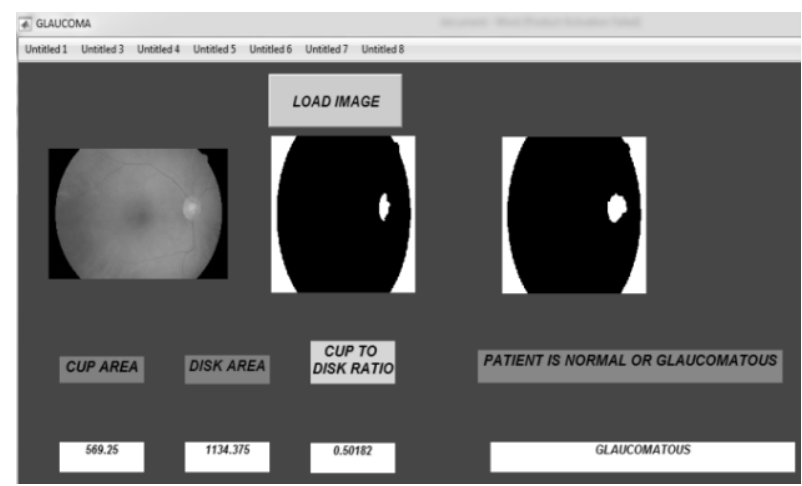

Fig. 10: GUI window displaying for glaucoma results

The outcomes Cup area, Disc area and Cup to Disc ratio are $569.25,1134.375$ and 0.5081 respectively. Artificial Neural Network is the class that epitomizes the neural system non linearity estimator [17]. The neural system will execute for 1000 epochs, estimates time, performance, mu and gradient values and is shown in Figure 11.

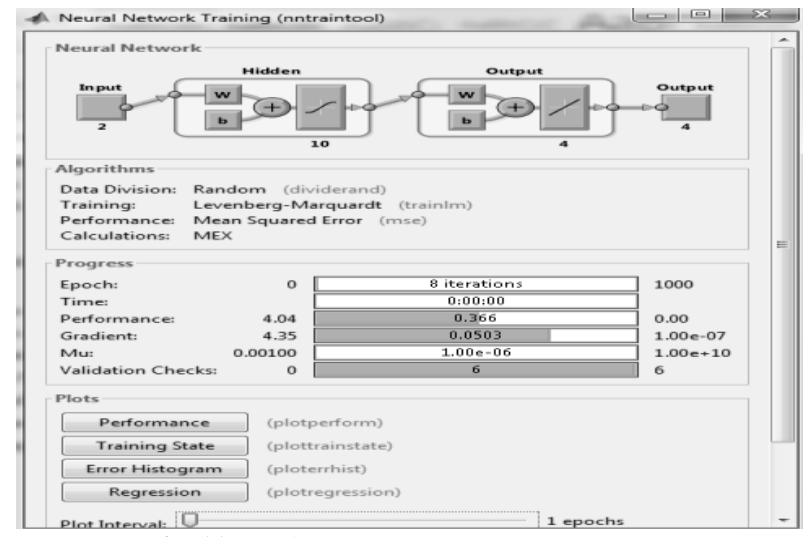

Fig. 11: Artificial neural networks architecture

The neural network performance plot is shown in Figure 12 . Whenever the training either stops or increases in validation error, the best performance is taken from the epoch with the lowest validation error[7]. Here the best validation performance has taken place at 2 epochs. 


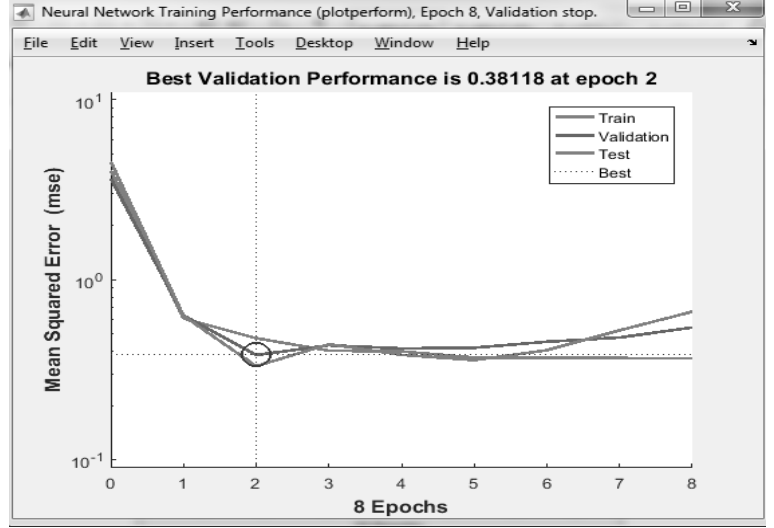

Fig. 12: Performance plot of ANN

The neural network training plot is displayed in Figure 13. Plots the training state from a training record TR returned by train.

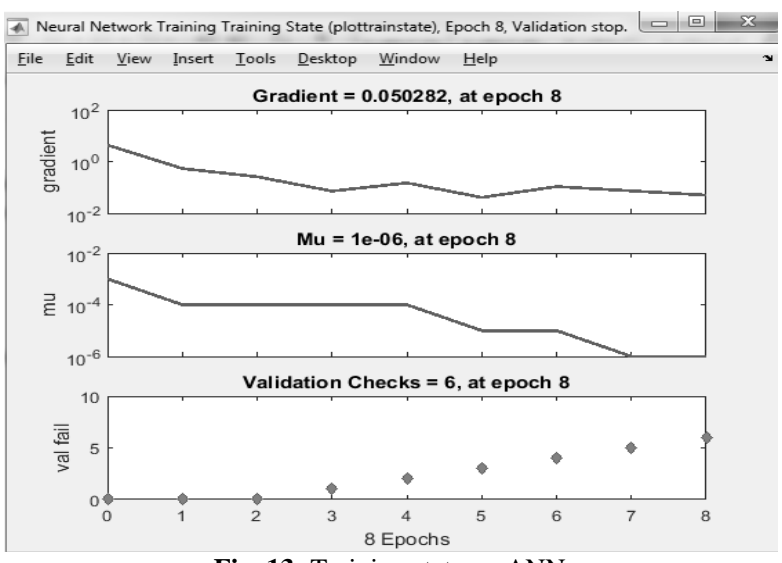

Fig. 13: Training state on ANN

The neural network training plot as displayed in Figure 14. Plot a histogram of error values from targets and outputs. The blue, green and red bars signify training data, validation data and testing data respectively.

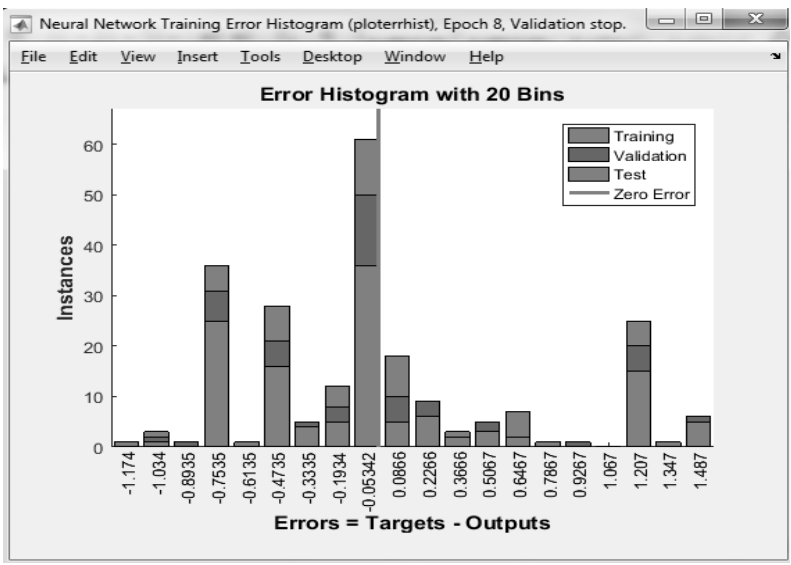

Fig. 14: Error histogram plot of ANN

The training, validation, testing data and overall are appeared in Figure 15. The dashed line represents the distinction between the output and perfect results outcomes which is equivalent to the targets. The solid line represents to the best fit straight line amongst yields or outputs and targets[12].

ANN groups the CTD ratios into four classifications it is appeared in Figure 16. The ANN groups the disease as Normal or typical, first stage, second stage and third or advanced stage [20].

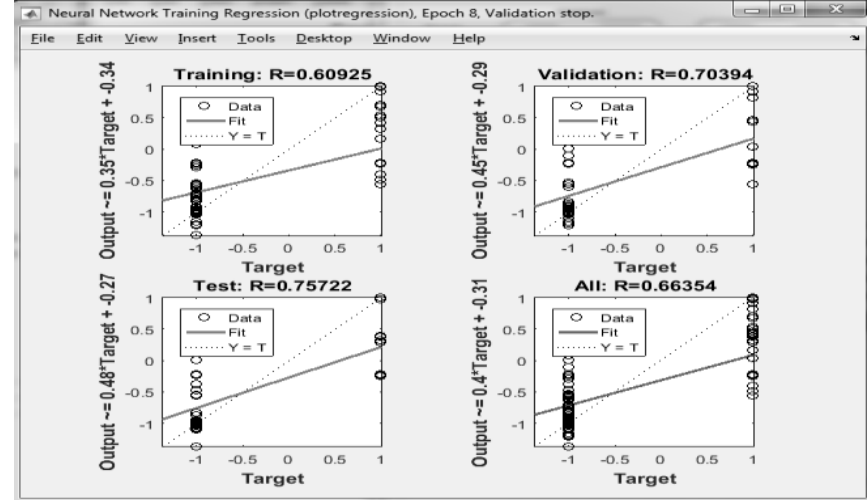

Fig. 15: Error histogram plot of ANN

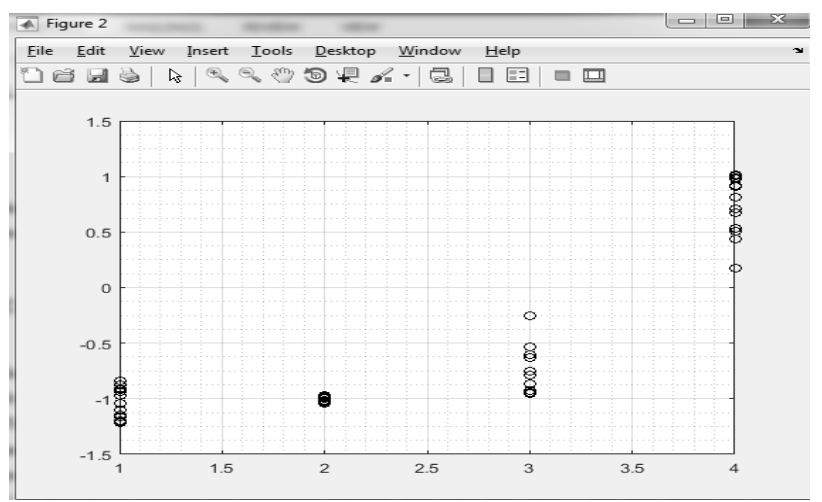

Fig. 16: ANN classification output

\section{Conclusion}

In the proposed research work an algorithmic approach is implemented to glaucoma identification by extracting its energy features from the data base of fundus images and the areas of the optic disc and optic cup from the retinal fundus images. The CTD proportions filled in as contributions to neural system for the grouping of various four classes which demonstrates accurate classification. Counterfeit Neural Networks plots the performance, error histogram and regression plots are plotted. The proposed system exhibits better accuracy compared to existing glaucoma classification systems. This system is cost effective and can be readily used in the hospitals. This system reduces doctor's burden and overcomes human error. In future the system can be incorporated with artificial intelligence to classify other abnormalities of the eye as well. It can also be designed to generate a report by itself with full information of the patient so that it can be used for telemedicine purpose.

\section{References}

[1] N. Annu ,\& J. Justin , (2013) "Classification of Glaucoma Images using Wavelet based Energy Features and PCA", International Journal of Scientific \& Engineering Research, Vol.5, No.2, ISSN: 0975-4024, 2013.

[2] U. R. Acharya, S. Dua , S.VinithaSree\& Chua C.K. Chua, "Automated diagnosis of glaucoma using texture and higher order spectra features", Information Technology in Biomedicine, IEEE Transactions on, 15(3), 449-455, 2011.

[3] D. Chaitali, S.B Dhumane\& P.G. Patil "Automated Glaucoma Detection using Cup to Disc Ratio", Vol. 4, Issue 7, ISSN(Online) : 2319-875, ISSN (Print) : 2347-6710, 2015.

[4] S. Dua, U.R. Acharya, P. Chowriappa , \&S.V.Sree, "Waveletbased energy features for glaucomatous image classification", Information Technology in Biomedicine, IEEE Transactions on, 16(1), pp 80-87,2012.

[5] R. Gayathri,, P.V. Rao, \& S. Aruna, "Automated glaucoma detection system based on wavelet energy features and ANN", International Conference in Advances in Computing, Communications and Informatics ( ICACCI), New Delhi, pp. 2808-2812, IEEE, 2014 
[6] R. Gayathri , S. Aruna , P.V. Rao , (2016) "A Novel Approach For Glaucoma Detection Using Cup To Disk Ratio", International Conference of Researches in Science, Management and Engineering, Journals of applied sciences and research,Coimbatore, page Nu 28-33.ISSN : 1816-157X, 2016.

[7] D.S. Grewal , R. Jain , P.S Grewal. \&Rihani , "Artificial neura network-based glaucoma diagnosis using retinal nerve fibre laye analysis", European Journal of Ophthalmology , Vol. 18 no. 6, pp. 915-921,2008

[8] KurnikaChoudhary, ShamikTiwari, "ANN Glaucoma Detection using Cup-to-Disk Ratio and Neuroretinal Rim”, International Journal of Computer Applications (0975 - 8887) Volume 111 No $11,2015$.

[9] C. H. Li, B.C Kuo , C.T. Lin \& C. Huang , "A spatialcontextual support vector machine for remotely sensed image classification", Geoscience and Remote Sensing, IEEE Transactions on, 50(3), 784-799, 2012

[10] F. Mianji\& Y. Zhang, (2011) "SVM-based unmixing- toclassification conversion for hyper spectral abundance quantification. Geoscience and Remote Sensing", IEEE Transactions on, 49(11), 4318-4327, 2011.

[11] Nidhi Shah, NarendraLimbad, "A Literature Survey on Glaucoma Detection Techniques using Fundus Images", IJSRD - Vol. 2, Issue 09, ISSN (online): 2321-0613, 2014.

[12] Parveen Kumar, Pooja Sharma, "Artificial Neural Networks-A Study", International Journal of Emerging Engineering Research and Technology, Volume 2, Issue 2, PP 143-148, 2014.

[13] PoojaChaudhari , A. GirishKulkarni, "Using Artificial Neural Network to Detect Glaucoma with the Help of Cup to Disk Ratio", IJARECEVolume5,Issue7, page no 1967, 2016.

[14] G. Quellec , S.R Russell, \& M.D. Abràmoff , “Optimal filter framework for automated, instantaneous detection of lesions in retinal images", Medical Imaging, IEEE Transactions on, 30(2), 523-533, 2011.

[15] P.V. Rao ,R. Gayathri\& R. Sunitha ,"A Novel Approach for Design and Analysis of Diabetic Retinopathy Glaucoma Detection using Cup to Disk Ration and ANN", 2nd International Conference on Nanomaterials and Technologies(CNT),Hyderabad, pp. 2211-8128, Published by Elsevier.2014.

[16] R. Raghul ,S. Lakshmi, "A NEW APPROACH FOR DETECTION OF BLOOD VESSEL TRACKING SYSTEM AND GLAUCOMA FOR DIABETIC PATIENT", VOL. 11, NO. 11, ISSN 1819-6608, 2016.

[17] O. Sheeba ,Jithin George, P.K. Rajin , and SherinGeorge,"Glaucoma Detection Using Artificial Neural Network”, IACSIT, Vol. 6, No. 2. DOI: 10.7763/IJET.2014.V6. $687,2014$.

[18] L. Shen\& S. Jia (2011), “ Three-dimensional Gabor wavelets for pixel-based hyperspectral imagery classification", IEEE Trans, on Geoscience And Remote Sensing, Vol. 49 , No 12, 5039 5046, 2011

[19] P.P Swapna and M.G. Mini, "A Regression Neural Network based Glaucoma Detection System using Texture Features", IJCCIE, Vol. 3, Issue 2, ISSN 2349-1469 EISSN 2349$1477,2016$.

[20] T. Vijayan, Ashutosh Singh, "Glaucoma Recognition and Segmentation Using Feed Forward Neural Network and Optical physics", International Journal of Advanced Research in Electrical, Electronics and Instrumentation Engineering, Vol. 4 Issue 4,2015.

[21] Gayathri R, DrRao.P.V, Aruna .S, "Glaucoma Detection System Based on Wavelet Energy Features , Cup to Disc Ratio, Threshold Value And ANN", Cashtech-2017 conference, VignanaBharathi Institute of Technology, page num 1277-1283, Telangana, Hyderabad, India

[22] T.Padmapriya, Ms. N. Dhivya, Ms U. Udhayamathi, "Minimizing Communication Cost In Wireless Sensor Networks To Avoid Packet Retransmission", International Innovative Research Journal of Engineering and Technology, Vol. 2, Special Issue, pp. 38-42.

[23] S.V.Manikanthan and K.srividhya "An Android based secure access control using ARM and cloud computing", Published in Electronics and Communication Systems (ICECS), 2015 2nd International Conference on 26-27 Feb. 2015,Publisher: IEEE,DOI: 10.1109/ECS.2015.7124833.

[24] Rajesh, M., and J. M. Gnanasekar. \&quot;Constructing WellOrganized Wireless Sensor Networks with Low-Leve Identification.\&quot; World Engineering \&amp; Applied Sciences Journal 7.1 (2016)
[25] S.V.Manikanthan and T.Padmapriya "Recent Trends In M2m Communications In 4g Networks And Evolution Towards 5g", International Journal of Pure and Applied Mathematics, ISSN NO:1314-3395, Vol-115, Issue -8, Sep 2017 